VOL. $2(1970), 432$.

\title{
Moving loads on elastic and thermoelastic solids
}

\author{
A. M. Roberts
}

The two dimensional disturbances generated by a steadily moving line load on the surface of an elastic half space have been investigated, both for steady state (coordinates moving with the load) and transient (motion starting at $t=0$ ) situations.

Steady state problems with mixed (stress-displacement) boundary conditions can be solved by adapting known Riemann-Hilbert complex variable techniques from static plane elasticity. For very fast load speeds the equations of motion become hyperbolic, and wave effects along characteristics of these equations are obtained. The sign of the vertical surface displacement is reversed if the load speed lies between the Rayleigh wave speed and the dilatational wave speed.

This anomalous surface displacement is clarified by the solution of the transient problem for a moving delta function load. Results are obtained from Fourier and Laplace transforms and a Cagniard inversion. It is seen that the vertical displacement is downward for the Rayleigh pulse, but upward beneath the load in the above speed range.

The corresponding two dimensional coupled thermoelastic problems for delta function loads are considered using integral transform techniques. In the steady state situation, physical variables are reduced to single integrals which can be estimated asymptotically for large distances and load speed. The transient problem leads to double inversion integrals which again may be estimated asymptotically. It is found that the thermal coupling damps both the compressional and Rayleigh waves and also completely blurs the reflected (rotational) 'head' waves coming from the stress free surface.

Received 24 October 1969. Thesis submitted to the University of Melbourne, March 1969. Degree approved, October 1969. Supervisors: Professor J.W. Craggs, Acting Professor W.W. Wood. 